\title{
THE RESEARCH CONFERENCE ON THE THEORY OF NUMBERS IN PASADENA
}

A Research Conference on the Theory of Numbers was held at the California Institute of Technology from June 22 to June 24, 1955 in conjunction with the thirty-sixth Annual Meeting of the Pacific Division of the American Association for the Advancement of Science. It was supported by a grant from the National Science Foundation to the California Institute of Technology. The members of the organizing committee were Sarvadaman Chowla, University of Colorado, Hans Rademacher, University of Pennsylvania, J. B. Rosser, Cornell University, J. T. Tate, Harvard University, Morgan Ward, California Institute of Technology, A. L. Whiteman (chairman), University of Southern California. Professor H. F. Bohnenblust of the California Institute of Technology was the local director of arrangements. The Conference was open to all interested mathematicians, and the total attendance was approximately seventy-five.

Through the courtesy of Caltech and the A.A.A.S. hotel accommodations, lounges, recreational and dining facilities were made available to the Conference participants. A banquet was held at Taix French Restaurant on Thursday evening, June 23. On Friday afternoon a group photograph of those attending the meeting was taken in front of Throop Hall.

The program of the Conference consisted of twenty-four invited addresses. Fifteen of the speakers received subsidization from the National Science Foundation. There were six sessions presided over respectively by Professors J. B. Rosser, Morgan Ward, Sarvadaman Chowla, Emil Artin, Richard Brauer, and D. H. Lehmer.

Bound sets of abstracts of most of the papers on the program were circulated to all participants at the beginning of the Conference. It was felt that the value of the Conference was thereby greatly enhanced. The papers were presented in the following order with Dr. Joseph Lehner's paper being read by title.

Ernst G. Straus: The arithmetic of analytic functions.

Olga Taussky Todd: Matrix methods in algebraic number theory.

Morgan Ward: Divisibility sequences.

Emma Lehmer: On the location of Gauss' sums.

Albert Leon Whiteman: A sum connected with the partition function.

Tom M. Apostol: The approximate functional equation of Hecke's Dirichlet series. 
Richard Bellman: The generalized theta-functions of Hecke, Siegel and Maass.

Joseph Lehner: Partial-fraction decompositions and expansions of zero.

Lowell Schoenfeld: On the order of the zeta function near the line $\sigma=1$.

Atle Selberg: Discontinuous groups and harmonic analysis with applications to Dirichlet series and modular forms.

D. H. Lehmer: New results about Ramanujan's tau function.

P. T. Bateman: General properties of partition functions.

Richard Brauer: Number-theoretical investigations on groups of finite order.

J. T. Tate: Cohomology and class-field theory.

N. C. Ankeny: Universal zeta functions.

H. S. Vandiver: Fermat's last theorem.

Gordon Pall: Simultaneous representation by adjoint quadratic forms.

Marshall Hall, Jr.: The minima of binary quadratic forms.

Harvey Cohn: Accessibility of algebraic numbers with rounded norms.

Emil Artin: The classical finite simple groups and their orders.

J. Barkley Rosser: Some new extensions of Brun's method.

Sarvadaman Chowla: Remarks on Bernoulli numbers.

Ivan Niven: Normal numbers.

Alfred T. Brauer: The Schnirelman density of the sum of two sequences of which one has positive density.

The Conference members expressed regret that Professor Paul Erdös, who had been chosen to be the first speaker at the Conference, was unable to attend. The Conference members strongly supported the request being made by the American Mathematical Society that Professor Erdös be permitted to reenter the United States of America.

A resolution of thanks and appreciation to the California Institute of Technology was read by Professor D. H. Lehmer and unanimously approved.

By fostering open discussions the Conference proved to be a valuable means of disseminating information and ideas about fields in which vigorous advances are being made. The inspiration provided by the informal contacts between the students and the masters will unquestionably serve as an impetus for future important research.

Albert Leon Whiteman 